\title{
Morbidité et mortalité des enfants au service de pédiatrie de l'hôpital Nianankoro Fomba de Ségou
}

Morbidity and mortality of children at the pediatrics department of Nianankoro Fomba hospital of Segou

Bah $A^{1}$, Bagayoko $T^{2}$, Kassogué $A^{1}$, Dramé $B M^{3}$, Haber $B^{1}$, Thiéro $A^{1}$, Guindo $M^{1}$, Coulibaly $M^{1}$, SOW $S^{1}$, Koné SI ${ }^{4}$, Samaké $B^{5}$, Traoré $\mathrm{T}^{7}$, Keita $\mathrm{M}^{5}$, Coulibaly $\mathrm{SS}^{9}$, Coulibaly $\mathrm{A}^{8}$, Togo $\mathrm{MA}^{8}$, Fofana $\mathrm{A}^{10}$, Sanogo $\mathrm{A}^{6}$, Diallo $\mathrm{M}^{11}$, Diakité $\mathrm{L}^{12}$

\section{DOI : 10.53318/msp.v11i1.1898}

1. Service de Pédiatrie, Hôpital Nianankoro Fomba de Ségou, Mali (HNF, Ségou, Mali)

2. Service de médecine légale/travail, HNF, Ségou, Mali

3. Service de chirurgie Maxillo faciale, HNF, Ségou, Mali

4. Service d'urologie, HNF, Ségou, Mali

5. Service de chirurgie générale, HNF, Ségou, Mali

6. Service de cardiologie, HNF, Ségou, Mali

7. Service de gynécologie, HNF, Ségou, Mali

8. Service d'ophtalmologie, HNF, Ségou, Mali

9. Service de médecine générale, HNF, Ségou, Mali

10. Service d'otorhinolaryngologie, HNF, Ségou, Mal

11. Unité du Système d'Information Hospitalière, HNF, Ségou, Mali

12. Centre de santé de référence Famory Doumbia de Ségou (Mali)

Auteur Correspondant : Dr Adama Bah, Service de pédiatrie de l'hôpital Nianankoro Fomba de Ségou. E-mail bah750@gmail.com

\section{RESUME :}

Introduction : les pays en développement sont confrontés à de nombreuses difficultés dans le domaine de la santé.. Objectif : Ce travail visait à étudier la morbidité et la mortalité des enfants de 1 mois à 15 ans hospitalisés dans le service de pédiatrie de l'hôpital Nianankoro Fomba de Ségou. Matériel et Méthodes : Notre étude s'est déroulée du 1er janvier au 31 décembre 2016 dans le service de Pédiatrie de l'Hôpital Nianankoro Fomba. II s'agissait d'une étude rétrospective, descriptive. Ont été inclus tous les enfants de 1 mois à 15 ans hospitalisés. Résultats : Nous avons inclus 862 enfants de 1 mois à 15 ans. 70,10\% des patients avaient moins de 5 ans. Les garçons représentaient $60,30 \%$. La majorité des patients $(75,90 \%)$ résidaient dans le district sanitaire de Ségou. Les principaux diagnostics retrouvés étaient le paludisme $(53,20 \%)$, la malnutrition aiguë sévère avec complications $(17,90 \%)$ et les infections respiratoires aiguës $(10,20 \%)$. Les patients guéris représentaient $86,08 \%$ et le taux de décès était de 6,61\%.La majorité des patients décédés était des garçons $(57,89 \%)$. Les trois principales causes de décès étaient le paludisme grave $(42,10 \%)$, la malnutrition aiguë sévère avec complications $(35,09 \%)$ et les sepsis sévères $(7,02 \%)$ et les décès concernaient les enfants de moins de 5 ans $(71,93 \%)$. Conclusion : La morbidité et la mortalité en pédiatrie restent préoccupantes dans nos structures malgré les efforts. Cependant les causes sont évitables par le changement de comportement à tous les niveaux. Mots Clés : morbidité, mortalité, enfants, Ségou.

\section{ABSTRACT:}

Introduction: Developing countries face many challenges in the area of health. Objective: This work aimed to study the morbidity and mortality of children from 1 month to 15 years hospitalized in the pediatric department of the Nianankoro Fomba hospital in Segou. Material and Methods: Our study took place from January 1 to December 31, 2016 in the Pediatric Department of the Nianankoro Fomba Hospital. It was a retrospective, descriptive study. All hospitalized children from 1 month to 15 years were included. Results: We included 862 children from 1 month to 15 years. $70.10 \%$ of patients were under 5 years of age. Boys accounted for $60.30 \%$. The majority of patients $(75.90 \%)$ resided in the Segou health district. The main diagnoses found were malaria $(53.20 \%)$, severe acute malnutrition with complications (17.90\%) and acute respiratory infections (10.20\%). Cured patients accounted for $86.08 \%$ and the death rate was $6.61 \%$. The majority of the patients who died were boys (57.89\%). The three main causes of death were severe malaria $(42.10 \%)$, severe acute malnutrition with complications (35.09\%) and severe sepsis $(7.02 \%)$ and deaths were among children under 5 years of age (71.93\%). Conclusion: Morbidity and mortality in pediatrics remain a concern in our structures despite efforts. However, the causes are preventable by behavioural change at all levels.

Key words: Morbidity, Mortality, Children, Segou.

\section{Introduction :}

Les pays en développement sont confrontés à de nombreuses difficultés dans le domaine de la santé. Selon L'Organisation Mondiale de la Santé (OMS), en 2019, on estime que 5,2 millions d'enfants âgés de moins de 5 ans sont morts le plus souvent de maladies qui pourraient être évitées ou traitées. Les enfants âgés de 1 à 11 mois représentaient 1,5 million de ces décès, tandis que les enfants âgés de 1 à 4 ans représentaient 1,3 million de décès. Les nouveau-nés (moins de 28 jours) représentaient les 2,4 millions de décès restant. 500000 enfants plus âgés (5 à 9 ans) sont décédés en 2019. [1] Les enfants plus âgés (5-9 ans) ont connu l'une des plus fortes baisses de mortalité depuis 1990 (61\%), en raison d'une baisse des maladies infectieuses. Les traumatismes (y compris ceux dus aux accidents de la route et les noyades) sont les principales causes de décès chez les enfants plus âgés. [1] Si à l'échelle mondiale les progrès se sont accélérés en matière de réduction du taux de mortalité des moins de 5 ans, il existe une différence entre la mortalité des enfants de moins de 5 ans entre les régions et les pays. L'Afrique subsaharienne reste la région où le taux de mortalité des moins de 5 ans est le plus élevé au monde, avec un enfant sur 13 qui meurt avant son 5uième anniversaire, soit 20 ans de moins que 
la moyenne mondiale qui a atteint un taux de 1 sur 13 en 1999. [1]

Deux régions, l'Afrique subsaharienne et l'Asie centrale et méridionale, représentent plus de $80 \%$ des 5,2 millions de décès chez les enfants de moins de 5 ans en 2019, alors qu'elles ne représentent que $52 \%$ de la population mondiale des moins de 5 ans. [1]. Tout en notant des progrès dans l'amélioration des services de santé accordés aux enfants, il a été estimé que 60 millions d'enfants mourront avant l'âge de 5 ans d'ici 2030 si les inégalités ne sont pas réduites [1].

Au Mali selon l'EDS VI, pour la période des 5 années ayant précédé l'enquête, sur 1000 enfants nés vivants, 54 sont décédés avant leur premier anniversaire et sur 1000 naissances vivantes, 101 sont décédées avant d'atteindre leur cinquième anniversaire. Selon la même enquête, c'est dans les régions de Ségou, Kayes, Tombouctou et Mopti que les enfants de moins de 5 ans courent les risques les plus élevés de décéder avec des taux égaux ou supérieurs à $130 \%$ [2].

Selon les précédentes études faites dans notre pays et dans certains pays limitrophes, plus de $70 \%$ des décès d'enfants sont dus à des infections qui pour la plupart pouvaient être évitées ou traitées [3,4]. L'objectif de cette étude était de décrire la morbidité et la mortalité infantile au service de pédiatrie de l'hôpital Nianankoro Fomba de Ségou pour mieux orienter les stratégies de prise en charge des patients.

\section{Matériel et méthodes :}

L'hôpital Nianankoro Fomba est une structure de 2ème référence. II abrite plusieurs services dont celui de la pédiatrie. Le service de pédiatrie comprend trois unités : l'unité d'hospitalisation, l'unité de néonatologie et celle des urgences pédiatriques.

II s'agissait d'une étude rétrospective descriptive allant du 1 er Janvier au 31 Décembre 2016. Nous avons inclus tous les enfants âgés de 01 mois à 15 ans hospitalisés dans le service de pédiatrie. Des fiches d'exploitation préalablement élaborées ont été remplies à partir de dossiers médicaux. Les variables retenues étaient : l'âge, le sexe, la provenance, le motif de consultation, le mode d'admission, le niveau socioéconomique (la profession du père, l'âge et le niveau d'instruction des mères), le délai de consultation, l'heure et la période d'admission, la durée du séjour hospitalier, le diagnostic retenu, le devenir immédiat. Les données ont été saisies et analysées sur le Logiciel IBM SPSS Statistics version 20.

\section{Résultats :}

\section{Morbidité :}

Durant la période d'étude, 1246 enfants ont été hospitalisés au service de pédiatrie de l'hôpital Nianankoro Fomba. Nous avons inclus 862 enfants âgés de 1 mois à 15 ans dont $70,10 \%$ avaient moins de 5 ans. Le sexe masculin représentait $60,30 \%$ avec une sex ratio de 1,5 (Tableau I).Parmi nos patients, $75,90 \%$ résidaient dans le district sanitaire de Ségou (Tableau I). Ils étaient référés dans $42,80 \%$ des cas par les centres de santé communautaire, les centres de santé de référence de la région et les cabinets privés (Tableau I). Les principaux motifs de consultation étaient les convulsions $(21,80 \%)$, la pâleur $(18,20 \%)$, les diarrhées et les vomissements $(17,60 \%)$. Le délai de consultation était supérieur à 48 heures dans $83,30 \%$ des cas. Le pic des hospitalisations était obtenu en Août (16,70\%) (Figure 1). La durée d'hospitalisation dépassait 3 jours dans $68,30 \%$ des cas. Les principales pathologies rencontrées étaient : le paludisme grave (459 soit $53,20 \%$ ), la malnutrition aiguë sévère avec complications (154 soit $17,90 \%$ ) et les infections respiratoires aiguës (88 soit 10,20\%). Nous avons enregistré $82,30 \%$ de sorties autorisées.

Mortalité :

Parmi les 862 dossiers étudiés, nous avons enregistré 57 décès dont 41 chez les enfants de 1 à 59 mois $(71,93 \%$ des décès). Le taux de décès dans notre série était de $6,61 \%$ (Tableau II). Le sexe masculin a représenté $57,89 \%$ des décès. Parmi les patients décédés $70,18 \%$ résidaient dans le district sanitaire de Ségou et 38,59\% n'étaient pas référés. Nous avons notés 35 décès $(61,40 \%)$ chez les patients ayant une durée d'hospitalisation supérieure à 3 jours. Le paludisme grave $(42,10 \%)$, la malnutrition aiguë sévère avec complication $(35,09 \%)$ et les sepsis sévères $(7,02 \%)$ constituaient les principales causes de décès. Ces décès étaient plus fréquents au mois de Septembre $(24,56 \%)$.

\section{Discussion :}

La majorité des patients hospitalisés à la pédiatrie $(70,10 \%)$ avaient un âge compris entre 1 et 59 mois. Ce constat vient confirmer les résultats des études de Doumbia AK et al. [5], où les enfants de moins 5 ans représentaient $58 \%$.

Notre étude a montré une prédominance des hospitalisations chez les garçons. Les mêmes constats étaient retrouvées dans plusieurs études [3-6].

L'hôpital Nianankoro Fomba de Ségou est une structure de deuxième référence mais la majorité des patients consultait directement sans passer par les structures périphériques. Doumbia AK et al. avaient fait les mêmes remarques en 2013 [5]. Ceci montre une insuffisance ou un dysfonctionnement du système de référence et d'évacuation sanitaire dans la région de Ségou Le délai de consultation était long, $83,50 \%$ des enfants ont été admis plus de 2 jours après le début de la maladie. Doumbia AK et al. et Coulibaly A avaient trouvé $44 \%$ et $57 \%$ [5,7]. II existe une forte affluence aux mois d'Août Septembre et d'Octobre $(16,70 \% ; 15,40 \% ; 13,90 \%)$ qui correspondent à la période de forte transmission du paludisme. Malgré les campagnes de chimio prévention chez les enfants de 3 mois à 59 mois dans les districts sanitaires, le paludisme $(53,20 \%)$ était la première cause d'hospitalisation et était surtout fréquent chez les enfants de moins de 59 moins $(61,22 \%)$. Malgré l'absence d'Unité de Récupération et d'Education Nutritionnelle Intensive (URENI) appropriée la malnutrition aiguë sévère avec complications constituait la 
deuxième cause d'hospitalisation avec une fréquence de $17,90 \%$. Nos résultats se rapprochent de ceux de Doumbia AK et al. et de Kanté [5,8].Les infections respiratoires aiguës étaient également une préoccupation majeure $(10,20 \%)$. Les patients atteints d'infection respiratoire aiguë étaient généralement admis dans un tableau de détresse respiratoire sévère nécessitant souvent des mesures appropriées de réanimation. La déshydratation par diarrhée $(4,8 \%)$ était surtout fréquente chez les nourrissons de moins d'un an (52,50\%). Doumbia AK et al. avaient fait les mêmes constats [5].

Au cours de cette étude, le taux de décès était estimé à $6,60 \%$. Ce taux de décès était particulièrement élevé chez les enfants de moins de 5 ans $(71,93 \%)$ et parmi ceux-ci, $31,70 \%$ avaient moins d'un an. Les mêmes tendances pour les nourrissons de moins 12 mois se retrouvaient dans les études de Doumbia AK et al. (40\%) et de Coulibaly A (27\%) [5,7].Malgré que les infections respiratoires aiguës aient constitué la 3ème cause d'hospitalisation ; les principales pathologies qui ont constitué les trois premières causes de décès $(84,21 \%)$ étaient le paludisme grave, la malnutrition aiguë sévère avec complications et les sepsis sévères. Doumbia AK et al. dans une étude réalisée au CHU Gabriel Touré, avaient trouvé que les trois premières causes de décès chez les enfants de moins de 5ans étaient le paludisme grave, la malnutrition aiguë sévère avec complications et les infections respiratoires aiguës (98\%) [5]. Au Maroc, la mortalité post-natale est dominée par les pathologies infectieuses $(49,55 \%)$ notamment les pneumonies (33\%), suivies des cardiopathies $(10,42 \%)$ [9].

Les patients qui décédaient avant la 72ème heure de leur hospitalisation représentaient $38,60 \%$. Le retard de consultation et de diagnostic liés à l'insuffisance du plateau technique peut expliquer cette brièveté de séjours hospitaliers. Le faible revenu des ménages et faible niveau d'instruction des mères sont des facteurs qui peuvent augmenter significativement la morbidité et la mortalité et peuvent aussi expliquer le retard à la consultation et la précocité des décès dans notre série. Le plus fort taux de décès a été enregistré au mois de septembre (soit $24,56 \%$ ), période de forte transmission du paludisme. La prise en charge des formes grave de paludisme demande des moyens et techniques appropriés de réanimation. Notre service ne dispose pas d'unité de soins intensifs bien équipe. Certains études ont mis en évidence d'autres aspects de la morbi-mortalité des enfants [10-12].

Limites de l'étude : Comme pour toute étude rétrospective, le recueil des données n'a pas été exhaustif du fait des informations manquantes dans certains dossiers clinique. Malgré ces limites, nous avons pu apprécier les principales caractéristiques de morbidité et de mortalité des enfants hospitalisés.

\section{Conclusion :}

La morbidité et la mortalité hospitalières chez les enfants restent dominées par le paludisme, la malnutrition aiguë sévère avec complications et les infections respiratoires aiguës. Ces résultats montrent qu'il faudrait améliorer la référence- évacuation dans notre région et équiper l'unité de soins intensifs du service de pédiatrie de l'hôpital Nianankoro Fomba pour réduire les décès des enfants.

\section{Références :}

1. OMS. Enfants : améliorer leur survie et leur bien-être. 9 septembre 2020.

https://www.who.int/fr/news-room/factsheets/detail/children-reducing-mortality

2-République du Mali : Enquête Démographique et de Santé 2018

3-Simpara B. Morbidité et mortalité des nourrissons hospitalisés dans le service de Pédiatrie du CHU Gabriel Touré. Mémoire, Université du Mali, 2010.

4-Asse K.V et al. Mortalité pédiatrique en 2007 et 2008 à l'Hôpital Général d'Abobo (Abidjan/Côte d'Ivoire). http://saranf.net//MG/articlePDF/Mortalit-ped en 2007.

5-Doumbia AK, Togo B, Togo $P$, Traoré F, Coulibaly O, Dembélé A, Maïga B, Dicko F, Diakité AA, Sylla M. Morbidité et mortalité chez les enfants de 01 à 59 mois hospitalisés au service de pédiatrie générale du $\mathrm{CHU}$ Gabriel Touré de janvier à décembre 2013. Revue Malienne d'Infectiologie et de Microbiologie 2016, Tome 8, 54-62.

6-Coulibaly A. Morbidité et mortalité à l'unité de réanimation pédiatrique du CHU GT. A propos de 975 cas. Thèse de Doctorat, Université du Mali. Faculté de Médecine Bamako ; 2008.

7-Ghorbal F.S. Evolution de la morbidité et de la mortalité dans ' un service de pédiatrie générale : étude comparative entre l'année 1991 et l'année 2007. Life Sciences. Université de Tunis El-Mana, 2009. https://tel.archives-ouvertes.fr/tel00552828.

8-Kanté $\mathbf{M}$. Morbidité et mortalité infanto-juvénile au centre de sante de référence de la commune 5 du district de Bamako. Thèse de Doctorat, Université du Mali. Faculté de Médecine Bamako ; 2012.

9-Lasri N. Épidémiologie de la mortalité de l'enfant à l'hôpital mère-enfant ; Centre Hospitalier Universitaire Mohammed VI de Marrakech : étude rétrospective sur 5 ans. Thèse $N^{\circ} 147$. Université Cadi Ayyad. Faculté de Médecine et de Pharmacie Marrakech. Année 2015.

10- Buckowski A, Rose E. Pediatric stroke: diagnosis and management in the emergency department. Pediatr Emerg Med Pract [Internet]. nov 2019 [cité 10 août 2021];16(11). Disponible sur:

https://pubmed.ncbi.nlm.nih.gov/31647863/

11- Mendelson J. Emergency Department Management of Pediatric Shock. Emerg Med Clin North Am [Internet]. mai 2018 [cité 10 août 2021];36(2). Disponible sur: https://pubmed.ncbi.nlm.nih.gov/29622332/

12-Kramer RE, Lerner DG, Lin T, Manfredi M, Shah M, Stephen TC, et al. Management of ingested foreign bodies in children: a clinical report of the NASPGHAN Endoscopy Committee. J Pediatr Gastroenterol Nutr. avr 2015;60(4):562-74. 
Liste des tableaux et figures

Tableau II : Devenir des malades

\begin{tabular}{|l|c|c|}
\hline Devenir & Effectif & $\%$ \\
\hline Guérison & 742 & 86,08 \\
\hline Décès & 57 & 6,61 \\
\hline Evasion contre avis & 37 & 1,04 \\
\hline $\begin{array}{l}\text { Sortie } \\
\text { médical }\end{array}$ & 17,29 \\
\hline Evacuation & 17 & 1,98 \\
\hline
\end{tabular}

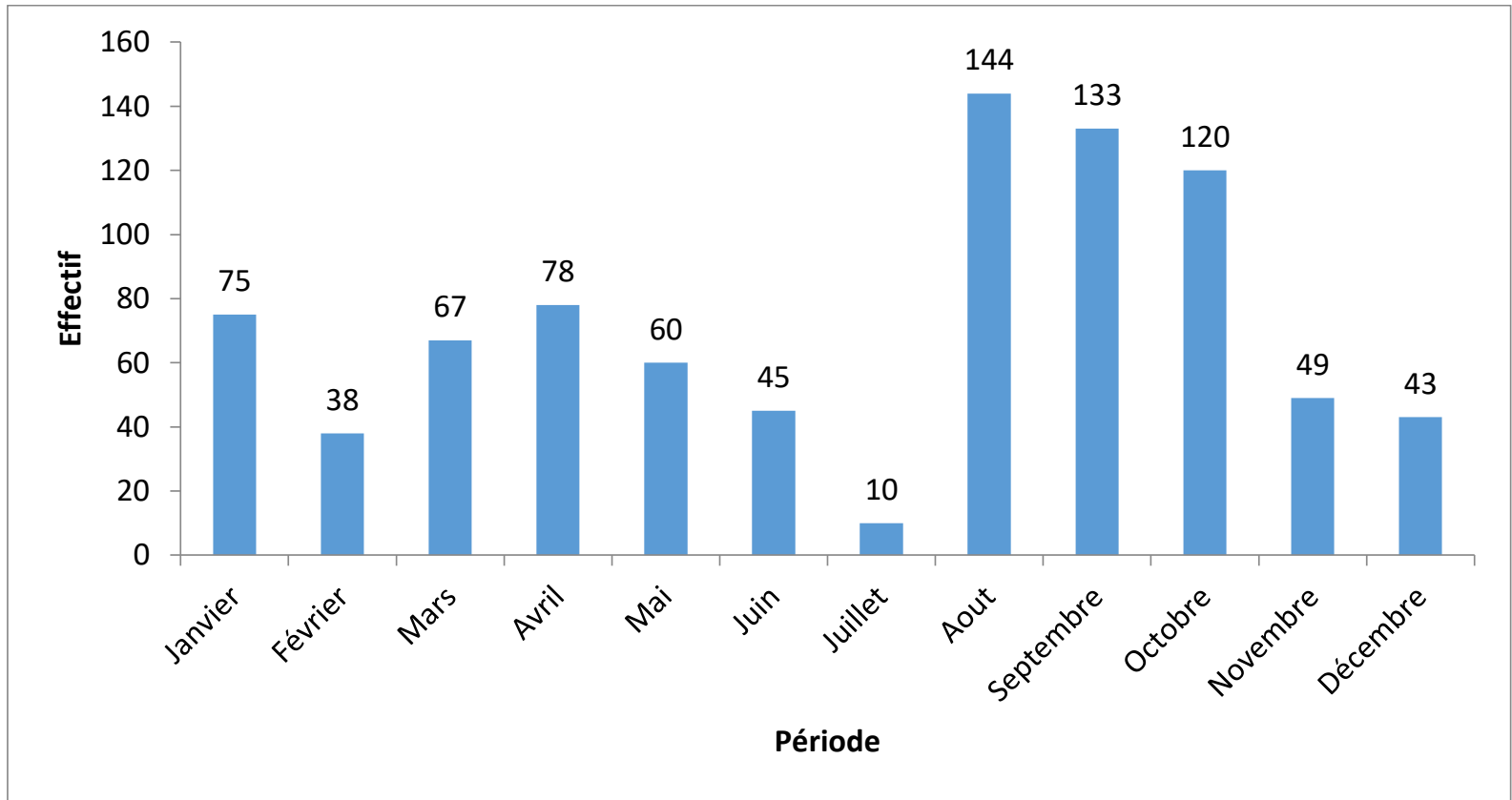

Figure 1 : Répartition des malades selon la période d'admission
Tableau I : Caractéristiques sociodémographiques des malades

\begin{tabular}{|l|l|l|}
\hline $\begin{array}{l}\text { Caractéristiques } \\
\text { sociodémographiques des } \\
\text { malades }\end{array}$ & Effectif & $\%$ \\
\hline Age & & \\
\hline $1-11$ mois & 178 & 20.6 \\
\hline $12-24$ mois & 250 & 29 \\
\hline $25-59$ mois & 180 & 20,9 \\
\hline Plus de 59 mois & 254 & 29,5 \\
\hline Sexe & & \\
\hline Masculin & 520 & 60,3 \\
\hline Féminin & 342 & 39,7 \\
\hline Mode d'admission & & \\
\hline Référé & 369 & 42,8 \\
\hline Non référé & 493 & 57,2 \\
\hline Heure d'admission & & \\
\hline $08-16$ heures & 497 & 57,7 \\
\hline $16-08$ heures & 365 & 42,3 \\
\hline Provenance & & \\
\hline District sanitaire de Ségou & 654 & 75,9 \\
\hline $\begin{array}{l}\text { Hors district sanitaire de } \\
\text { Ségou }\end{array}$ & 208 & 24,1 \\
\hline & & \\
\hline
\end{tabular}

Période 